\title{
Observation of three-mode parametric instability
}

\author{
X. Chen, * C. Zhao, S. Danilishin, L. Ju, and D. Blair \\ School of Physics, University of Western Australia, WA 6009, Australia
}

H. Wang
Department of Physics, Beijing Normal University, Beijing 100875, China

S. P. Vyatchanin

Faculty of Physics, M.V. Lomonosov Moscow State University, Moscow 119992, Russia

C. Molinelli, A. Kuhn, S. Gras, ${ }^{\dagger}$ T. Briant, P.-F. Cohadon, and A. Heidmann

Laboratoire Kastler Brossel, UPMC - Sorbonne Universités, CNRS, ENS - PSL Research University, Collège de France, F-75005 Paris, France

\author{
I. Roch-Jeune \\ Institut d'Electronique, de Microélectronique et de Nanotechnologie, CNRS UMR 8520, F-59652 Villeneuve d'Ascq, France \\ R. Flaminio, C. Michel, and L. Pinard \\ Laboratoire des Matériaux Avancés, CNRS/IN2P3, Université Claude Bernard - Lyon I, F-69100 Villeurbanne, France
}

(Received 22 October 2014; published 26 March 2015)

\begin{abstract}
Three-mode parametric interactions occur in triply resonant optomechanical systems: Photons from an optical pump mode are coherently scattered to a high-order mode by mechanical motion of the cavity mirrors, and these modes resonantly interact via radiation pressure force when some conditions are met. They can either pump energy into acoustic modes, leading to parametric instability, or extract mechanical energy, leading to optomechanical cooling. Such effects are predicted to occur in long baseline advanced gravitational-wave detectors, possibly jeopardizing their stable operation. We have demonstrated both three-mode cooling and amplification in two different three-mode optomechanical systems. We report an observation of the three-mode parametric instability in a free-space Fabry-Perot cavity, with ring-up amplitude saturation.
\end{abstract}

DOI: 10.1103/PhysRevA.91.033832

PACS number(s): 42.65.Yj, 42.50.Wk

\section{INTRODUCTION}

In 2001, Braginsky et al. predicted three-mode parametric instability in advanced gravitational-wave detectors [1,2], which would be caused by coincident frequency matching and mode shape matching between mirror mechanical modes and high-order optical modes. The high mechanical and optical mode density of these systems makes it likely that such interactions occur accidentally. Subsequently detailed modeling [3-5] verified the predictions and experimental tests on suspended optical cavities [6,7] demonstrated three-mode interactions below the instability threshold.

Three-mode instability has been observed in relatively low-quality factor solid-state resonators [8-12] and in a microwave system [13], but has not been reported in free-space optical cavities. The challenge can be met either by using very high optical power in large-scale optical cavities such as Advanced LIGO [14] or Advanced Virgo [15] detectors, or at low power in table-top cavities with suitable mode structure and a low-mass high-quality factor mechanical resonator.

In this paper we present two free-space cavity configurations suitable for investigating three-mode interactions and parametric instability. One is a cavity coupled to a silicon

\footnotetext{
*xu.chen@uwa.edu.au

${ }^{\dagger}$ Present address: LIGO Laboratory, MIT, Cambridge, USA.
}

bridge microresonator, very similar to one already used for two-mode cooling and instability experiments [16]. The second is a cavity with an intracavity moving membrane [17].

We first present a summary of the conventional theory of three-mode parametric interactions. We then describe the experiment with a bridge resonator, which allows us to demonstrate the resonant character of both amplification and cooling processes, and the membrane experiment, which has demonstrated parametric instability in a free-space cavity. The latter experimental results reveal that the instability does not lead to the loss of cavity lock and are in excellent agreement with the theoretical model [18]. The implications of our results for advanced gravitational-wave detectors are briefly discussed.

\section{THEORY OF THREE-MODE PARAMETRIC INTERACTIONS}

Figure 1 presents a cartoon of two- and three-mode interactions for the case of the Stokes mechanical amplification process. An incident laser (optical power $P_{\text {in }}$, angular frequency $\left.\omega_{0}=2 \pi c / \lambda\right)$ is scattered by a moving mirror (resonance frequency $\Omega_{\mathrm{m}} / 2 \pi$ ), creating two sidebands: Stokes (at $\omega_{0}-\Omega_{\mathrm{m}}$ ) and anti-Stokes $\left(\omega_{0}+\Omega_{\mathrm{m}}\right.$ ). In a cavity cooling experiment [19-21], the anti-Stokes band is favored by tuning the cavity close to $\omega_{0}+\Omega_{\mathrm{m}}$ to damp and effectively cool the resonator motion. Mechanical motion is amplified in the opposite Stokes case. 

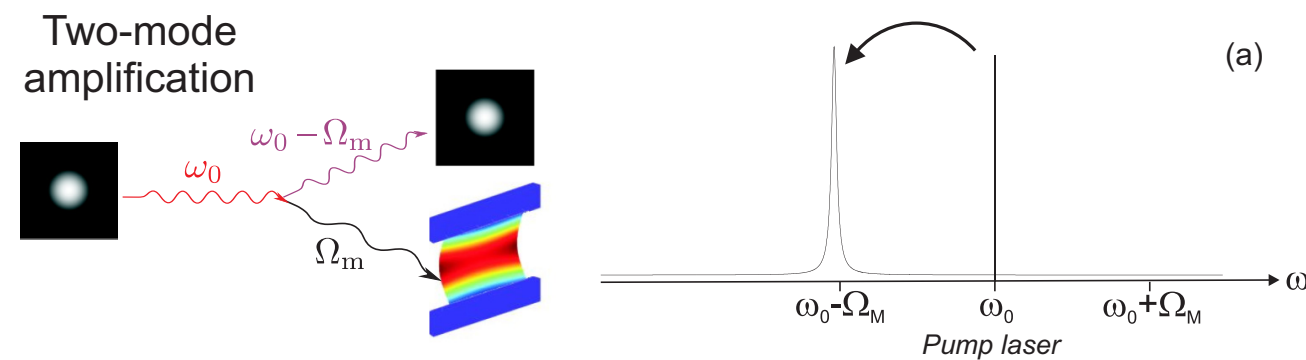

Stokes band Anti-Stokes band
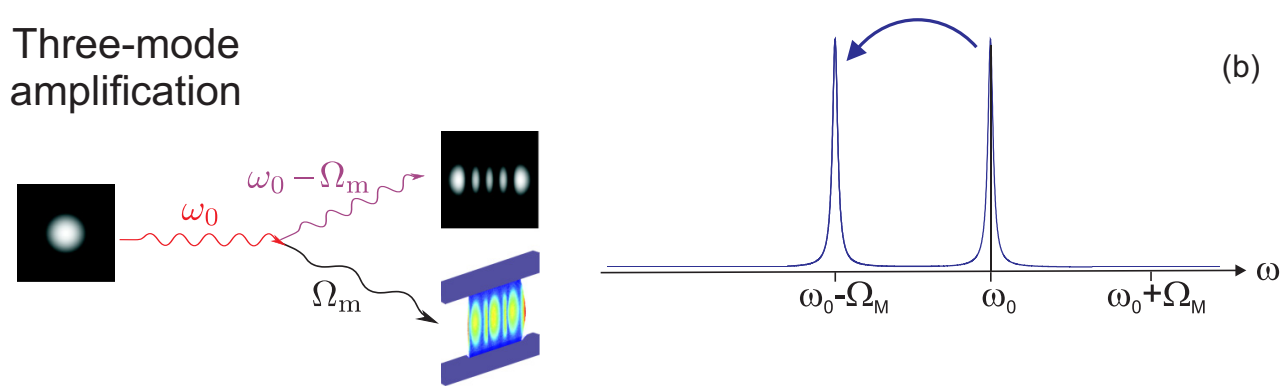

FIG. 1. (Color online) Radiation-pressure amplification seen as a macroscopic scattering process. (a) Two-mode amplification. The nonresonant incident photon at $\omega_{0}$ is scattered to the same transverse optical mode, with a frequency offset $\Omega_{\mathrm{m}}$ with respect to the incident photon. The cavity is detuned in order to favor the Stokes process, and the pump laser is far detuned from the resonance. (b) Three-mode amplification. The resonant photon is scattered to a different optical mode of the cavity. Both the pump laser and the Stokes band can be simultaneously resonant with the cavity. If the vibration profile of the mechanical mode matches the mode shape of the output optical mode, the system can achieve very strong coupling.

In three-mode systems, the mechanical mode of the moving mirror is coupled to not just one but two different optical cavity modes. The incident laser beam (pump mode $\omega_{0}=\omega_{\mathrm{p}}$ ) and the Stokes sideband can then be simultaneously resonant with the cavity (target higher-order mode $\omega_{\mathrm{s}}$ ), while still keeping the anti-Stokes band far detuned. In this situation, amplification appears as a scattering process of laser photons to a lower-frequency mode by mechanical phonon creation. The maximum efficiency is obtained when the laser is resonant with the pump mode and when the frequency offset $\Delta \omega=\omega_{\mathrm{s}}-\omega_{\mathrm{p}}$ between the target mode and the pump is close to $-\Omega_{\mathrm{m}}$, as shown in Fig. 1(b).

Under such conditions, the effective damping rate $\Gamma_{\text {eff }}$ of the mechanical resonator (mass $m$, mechanical quality factor $Q$, and intrinsic damping $\Gamma_{\mathrm{m}}=\Omega_{\mathrm{m}} / Q$ ) is related to the parametric gain $\mathcal{R}$ which fully characterizes the three-mode parametric interactions:

$$
\frac{\Gamma_{\text {eff }}}{\Gamma_{\mathrm{m}}}=1-\mathcal{R} .
$$

The parametric gain is given by

$$
\mathcal{R}=\mathcal{R}_{+}-\mathcal{R}_{-},
$$

where

$$
\mathcal{R}_{+}=\frac{16}{\pi \lambda c} \frac{\mathcal{F}_{\mathrm{p}} \mathcal{F}_{\mathrm{t}} Q P_{\text {in }} \Lambda}{m \Omega_{\mathrm{m}}^{2}}\left(\frac{1}{1+\left(\omega_{\mathrm{p}}-\Omega_{\mathrm{m}}-\omega_{\mathrm{s}}\right)^{2} / \Omega_{\mathrm{cav}}^{2}}\right)
$$

is the gain for the Stokes process and

$$
\mathcal{R}_{-}=\frac{16}{\pi \lambda c} \frac{\mathcal{F}_{\mathrm{p}} \mathcal{F}_{\mathrm{t}} Q P_{\text {in }} \Lambda}{m \Omega_{\mathrm{m}}^{2}}\left(\frac{1}{1+\left(\omega_{\mathrm{p}}+\Omega_{\mathrm{m}}-\omega_{\mathrm{s}}\right)^{2} / \Omega_{\text {cav }}^{2}}\right),
$$

is the gain for the anti-Stokes process. $\mathcal{F}_{\mathrm{p}}$ and $\mathcal{F}_{\mathrm{t}}$ are the optical finesses for the pump and target modes of the cavity, $\Omega_{\text {cav }} / 2 \pi$ the cavity bandwidth of the scattered mode, and $\Lambda$ the spatial overlap between mechanical mode $u$ and optical modes $v_{\mathrm{p}}$ and $v_{\mathrm{s}} \cdot \mathcal{R}$ is negative for cooling, positive for amplification and larger than 1 for instability [1].

If the power is enough to exceed the parametric instability threshold:

$$
P_{\mathrm{th}}=\frac{\hbar \omega_{\mathrm{p}} \gamma_{\mathrm{p}} \gamma_{\mathrm{s}} \Gamma_{\mathrm{m}}}{2 G_{\mathrm{ps}}^{2}}
$$

then the amplitude will grow exponentially with the rate,

$$
\Gamma \approx \frac{2 G_{\mathrm{ps}}^{2} P_{\mathrm{in}}}{\hbar \omega_{\mathrm{p}} \gamma_{p} \gamma_{\mathrm{s}}}-\gamma_{\mathrm{m}},
$$

where $G_{\mathrm{ps}}$ is the coupling strength.

When the loss of pump mode power through scattering into high-order mode becomes large, the amplitudes will reach their steady-state values:

$$
\begin{gathered}
\left|a_{\mathrm{p}}\right|^{2}=\frac{\Gamma_{\mathrm{m}} \gamma_{\mathrm{s}}}{G_{\mathrm{ps}}^{2}}, \\
\left|a_{\mathrm{s}}\right|^{2}=\frac{\Gamma_{\mathrm{m}}}{\gamma_{\mathrm{s}}}\left|b_{\mathrm{m}}\right|^{2}, \\
\left|b_{\mathrm{m}}\right|^{2}=-\frac{\gamma_{\mathrm{p}} \gamma_{\mathrm{s}}}{G_{\mathrm{ps}}^{2}}+\sqrt{\frac{2 \gamma_{\mathrm{p}} \gamma_{\mathrm{s}}}{\Gamma_{\mathrm{m}} G_{\mathrm{ps}}^{2}} \frac{P_{\mathrm{in}}}{\hbar \omega_{\mathrm{p}}}} .
\end{gathered}
$$

Here, $\left|a_{\mathrm{p}}\right|$ is the amplitude of the pump optical mode, $\left|a_{\mathrm{s}}\right|$ is the amplitude of the scattered target high-order mode, and $\left|b_{\mathrm{m}}\right|$ is 
the vibration amplitude of the mechanical mode of the moving mirror (see Appendices for a detailed derivation).

\section{DESIGN OF THE EXPERIMENTS}

To observe the three-mode interaction, two relevant optical modes have to be simultaneously resonant inside the cavity, while the frequency difference between the two optical modes must be tuned to the mechanical mode frequency to obtain maximum parametric gain [1]. The resonance frequencies of the mechanical modes of a typical table-top free-space optomechanical resonator span from a few hundreds of $\mathrm{kHz}$ to a few $\mathrm{MHz}$, while the free spectral ranges of centimeter-scale optical cavities usually lie in the $\mathrm{GHz}$ range. The two optical modes therefore need to be almost degenerate (compared with the scale of the free spectral range) and to be tuned with a relative precision of $\sim 10^{-6}$. This can be achieved with a careful choice of the mirror radii of curvature and of the cavity length.

The second great challenge in these experiments is to ensure a significant spatial overlap between the mechanical mode and the optical modes.

\section{A. Semiconfocal cavity with silicon resonator}

Our first experiment, carried out at Laboratoire Kastler Brossel in Paris, exactly corresponds to the setup first considered by Braginsky et al. [1]: a single-ended linear Fabry-Perot cavity with a moving end mirror, whose motion induces sidebands that can be amplified by the cavity. The moving mirror is a $1 \mathrm{~mm} \times 800 \mu \mathrm{m} \times 30 \mu \mathrm{m}$ silicon doubly clamped beam $[16,22]$. Such a micromirror has a number of mechanical modes with appropriate vibration profiles (see Fig. 4) and mechanical resonance frequencies close to $5 \mathrm{MHz}$.

The frequency resonance condition is reached with a cavity close to the semiconfocal configuration, with the moving mirror used as a nearly flat end mirror. The input mirror is concave, with a 50-mm radius of curvature. The resonance frequency offset $\Delta \omega / 2 \pi$ scales linearly with the cavity length $L$, with a measured rate of $250 \mathrm{kHz} / \mu \mathrm{m}$ close to the degeneracy point $L=R / 2$ between the $\mathrm{TEM}_{00}$ and $\mathrm{TEM}_{04}$ modes (see Fig. 2). The cavity has a finesse of 30000 for the TEM 00 pump mode, 24000 for the $\mathrm{TEM}_{04}$ scattered mode, with a corresponding cavity bandwidth $\Omega_{\text {cav }} / 2 \pi$ around $80 \mathrm{kHz}$.

Demonstration of three-mode interactions requires the tuning of the frequency difference $\Delta \omega$ with a precision that is small compared with the cavity bandwidth $\Omega_{\text {cav }}$. This requires the cavity length to be controlled at the 100-nm level. To change the cavity length, we have used both a stepper motor to set either the Stokes or the anti-Stokes band close to resonance, and a piezoelectric transducer for finer displacement tuning in the vicinity of the resonances.

\section{B. Near-confocal cavity with intracavity membrane}

To observe higher parametric gains, it is convenient to use a lower mass resonator. In our second experiment, performed at the University of Western Australia in Perth, we used a 50-nm silicon nitride membrane as the mechanical resonator (see Fig. 3). Such membranes have low optical absorption and can be embedded in high-finesse optical cavities, creating a
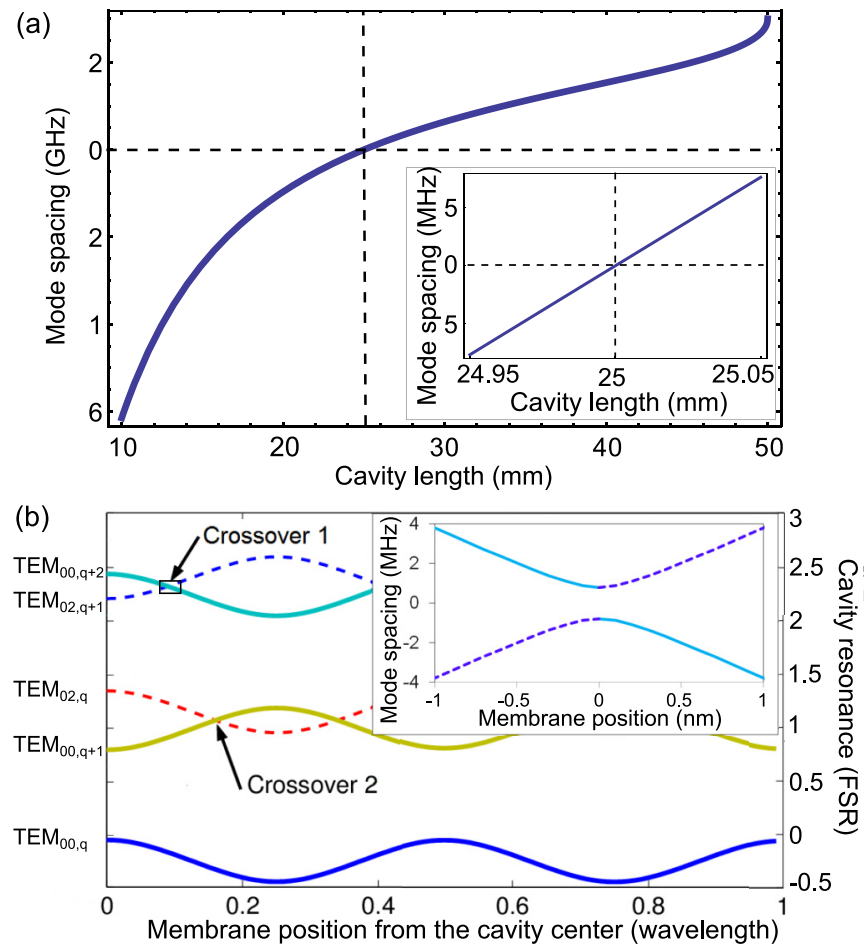

FIG. 2. (Color online) Cavity tuning close to the three-mode resonance condition, for the two experimental setups envisioned. (a) Mode spacing $\Delta \omega / 2 \pi$ for the case of a $\mathrm{TEM}_{00}$ and a $\mathrm{TEM}_{04}$ mode, as a function of cavity length. The curves are computed for a plano-concave cavity, with a radius of curvature $R=50 \mathrm{~mm}$. Insert shows a closeup of the degeneracy point. (b) Optical resonance frequencies of the compound cavity as a function of the membrane position $z_{0}$. For simplicity, only the relevant transverse modes $\mathrm{TEM}_{00}$ and $\mathrm{TEM}_{02}$ are shown. (Insert) Closeup of the avoided crossing near Crossover 1 (with relative membrane position). Note the different horizontal and vertical scales.
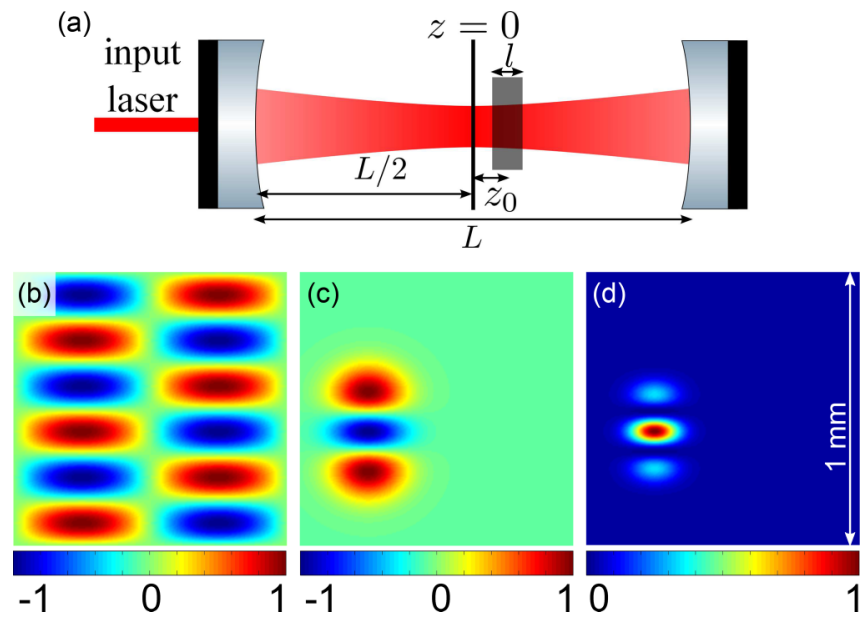

FIG. 3. (Color online) Membrane-in-the-middle configuration for the three-mode interaction experiment and illustration of the overlap between the membrane $(2,6)$ mechanical mode and $\mathrm{TEM}_{00}$ and $\mathrm{TEM}_{20}$ optical modes. (a) Cavity configuration. (b) Mode shape of the membrane $(2,6)$ mode. (c) Mode shape of the $\mathrm{TEM}_{20}$ cavity mode. (d) Product of the mode shapes, with the optical mode correctly located on the membrane for optimized overlap. 
coupled cavity configuration that can mimic a conventional setup. They also have high-quality factor mechanical modes in the $\mathrm{MHz}$ range [17]. The measured resonance frequency for the $(2,6)$ mode under investigation is $\Omega_{\mathrm{m}} / 2 \pi \sim 1.718 \mathrm{MHz}$, with a mechanical $Q$ of $\simeq 10^{6}$ in vacuum.

To demonstrate the parametric instability, the membrane position is tuned so that the two optical modes have a frequency difference $\Delta \omega=-\Omega_{\mathrm{m}}$. Figure 2 shows the frequency structure of the coupled cavity as a function of the membrane position. One can see that when the membrane is near Crossover 1 or 2 , the frequency difference between the $\mathrm{TEM}_{00}$ mode and the target $\mathrm{TEM}_{02}$ mode can be small enough to match the membrane mechanical frequency. Crossover $1\left(z_{0}=0.09 \lambda\right)$ is closer to a node of the electric field and should have lower optical loss due to membrane absorption [23] than Crossover $2\left(z_{0}=0.16 \lambda\right)$.

In principle, tuning the membrane position allows the mode spacing between the $\mathrm{TEM}_{00}$ and $\mathrm{TEM}_{20}$ modes to be tuned to arbitrarily small value to match the membrane mechanical resonance frequency. However in practice, the crossovers are avoided due to mode coupling [24,25]. Thus there is a minimum frequency spacing at the nominal crossing point as shown in the insert in Fig. 2. One of the greatest challenges in this experiment has been the adjustment of the membrane position and its careful alignment to allow the minimum frequency spacing to be smaller than the chosen mechanical mode frequency.

Figure 3 shows the mode shapes of the $(2,6)$ mechanical mode, the optical cavity $\mathrm{TEM}_{20}$ mode shape profile, and the product of all three profiles - the $(2,6)$ mode, and the TEM 00 and $\mathrm{TEM}_{20}$ optical modes. The optimized overlap factor $\sim 0.11$ is obtained when cavity modes are correctly positioned on the membrane at a specific location. The maximum finesse observed with the membrane inserted at the Crossover 1 position is $\sim 13000$.

\section{EXPERIMENTAL RESULTS}

We present in the following experimental results obtained with both setups. Additional information on the experimental setups, their characterization, as well as details on the experimental results can be found in the Appendices.

\section{A. Three-mode cooling and amplification with the semiconfocal cavity}

Experimental results for the anti-Stokes process are presented in Fig. 4. Here we have used the effective damping rate $\gamma_{\text {eff }}$ of the mechanical resonator as a measure of the three-mode coupling. Close to the anti-Stokes process resonance $(\Delta \omega=$ $+\Omega_{\mathrm{m}}$ ), the thermal noise spectrum is both widened (and reduced) as expected. Similar effects were already observed in two-mode cooling experiments [16]. Close to the resonance $\Delta \omega=\Omega_{\mathrm{m}}$, we observe a parametric gain $\mathcal{R} \simeq-0.72$.

Similar measurements have been carried out for the neighboring mechanical modes $(3,7)$ and $(5,5)$ of the moving mirror. These results are also presented in Fig. 4 . For all three modes, we find that the frequency detuning $\Delta \omega$ corresponding to the maximal damping effect matches the mechanical resonance frequency $\Omega_{\mathrm{m}}$, deduced from the thermal noise spectrum, and

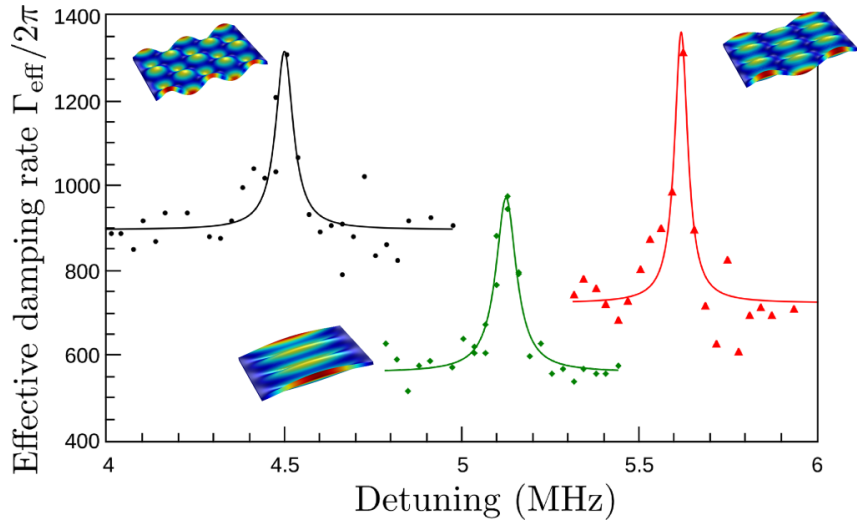

FIG. 4. (Color online) Experimental demonstration of the resonant character of the three-mode anti-Stokes process. Mechanical dampings of the $(1,7)$ [green (middle)], $(5,5)$ [black (left)], and $(3,7)$ [red (right)] mechanical modes. The damping increases substantially close to the three-mode resonance condition. Dots are experimental values of the effective damping, while the lines are fits to Eq. (4). Inserts display the simulated mode shape profiles of the mechanical modes.

the resulting damping of the mechanical mode. One can see that the damping returns to its intrinsic value outside the three-mode resonance. Note that different clamping losses result in different intrinsic damping values. Despite the large dispersion due to the low stability of the cavity, experimental points for all three mechanical modes are well fitted by Eq. (4), with a common optical bandwidth value close to $40 \mathrm{kHz}$, of the same order of magnitude as the cavity optical bandwidth $\Omega_{\text {cav }}$. Typical measured values for the absolute parametric gain are close to 0.5 .

Three-mode amplification has also been demonstrated close to the Stokes process resonance $\left(\Delta \omega=-\Omega_{\mathrm{m}}\right)$. As sweeping the length over $40 \mu \mathrm{m}$ to detune the cavity by $2 \Omega_{\mathrm{m}} / 2 \pi \simeq$ $10 \mathrm{MHz}$ also changes its alignment, the overlap $\Lambda$ and the observed parametric gain are reduced. Results are in good agreement with Eq. (3), and the measured maximal value for the gain in this case is $\mathcal{R}=0.41$ for mode $(1,7)$, for the same optical power as in the cooling experiment. In this experiment the parametric instability (gain $>1$ ) regime could not be achieved at the maximum usable input power of $5 \mathrm{~mW}$.

\section{B. Observation of three-mode parametric instability with the intracavity membrane}

We now describe the experiment performed with the membrane cavity. The cavity is first set close to the Stokes resonance condition. Once the laser is locked to the $\mathrm{TEM}_{00}$ mode and the optical mode is correctly positioned relative to the membrane acoustic mode, exponential ring-up of the mechanical $(2,6)$ mode occurs as soon as the input power exceeds the threshold of $R=1$, reaching saturation in a time of between 0.1 and $0.5 \mathrm{~s}$, as shown in Fig. 5. Saturation occurs without ruining the cavity locking. The figure shows that the saturation amplitude depends on the input power. Due to the very low-mass membrane and the high-finesse cavity used in this experiment, the threshold for parametric instability is expected to be only $\sim 3 \mu \mathrm{W}$. Hence very low powers must be 


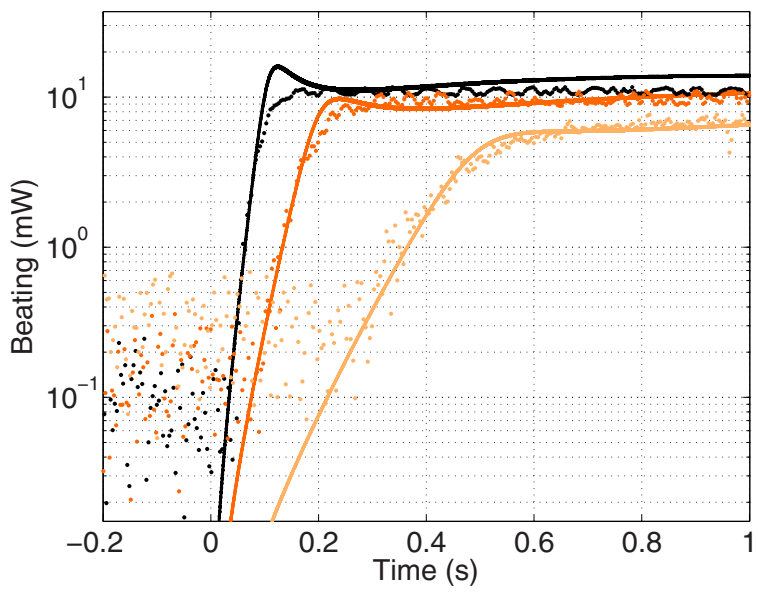

FIG. 5. (Color online) Experimental demonstration of the parametric instability. The dots are experimental data of the beatnote signal between the cavity $\mathrm{TEM}_{00}$ mode and $\mathrm{TEM}_{20}$ mode at mechanical frequency, as a function of time for different input powers. The curves display exponential ring-up before reaching saturation. The solid curves are simulation results for the corresponding input power. The input power is $26 \mu \mathrm{W}$ (black), $15 \mu \mathrm{W}$ [orange (dark gray)], and $7 \mu \mathrm{W}$ [yellow (light gray)].

used, which means that the beat-note signal between TEM $_{00}$ and $\mathrm{TEM}_{20}$ is initially below the photodetector noise floor.

Experimental results are presented in Fig. 5 for three different optical input powers: 7, 15, and $26 \mu \mathrm{W}$. The solid curves are simulations using the value of $G_{\mathrm{ps}}$ inferred from the fit performed in Fig. 6 and the same input powers. The measured ring-up times and beat-note steady-state amplitudes between the $\mathrm{TEM}_{00}$ and $\mathrm{TEM}_{20}$ modes are shown in Fig. 6 as a function of input power. Clearly there is good agreement between the experimental results and the large-amplitude model, except for some discrepancy at large input power.

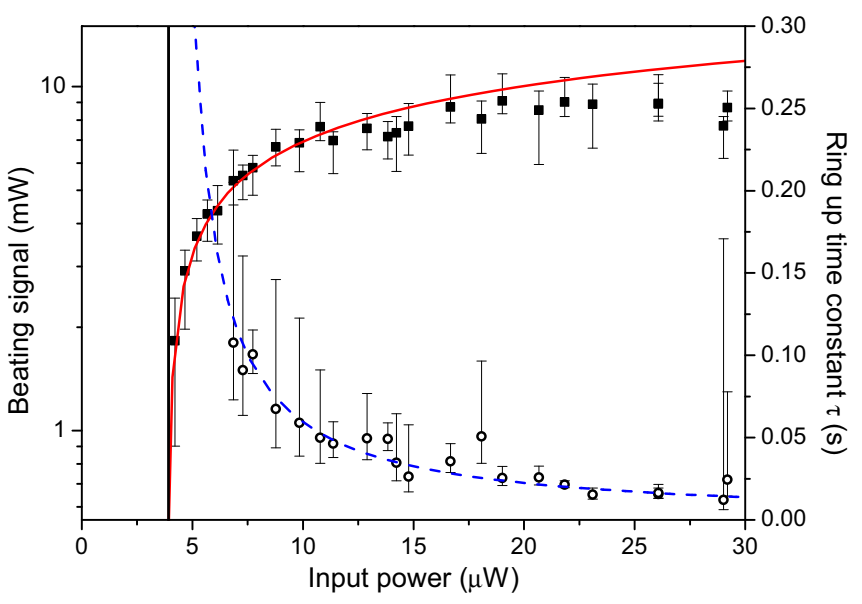

FIG. 6. (Color online) Measured steady-state beat-note signal amplitudes (solid squares) and ring-up times (hollow circles) as a function of the input power. The dashed blue line fit for the ring-up time is from Eq. (A2), and yields a value of $G_{\mathrm{ps}}=2 \pi \times 0.10 \mathrm{~Hz}$. The full red line fit for the beat-note amplitude is from Eqs. (A5) and (A6). The vertical line indicates the parametric instability threshold of $3.92 \mu \mathrm{W}$.
This could be due to thermal effects caused by the increased transverse mode intensity, which could lead to a decrease of the parametric gain. The fit for the ring-up time constant yields a value of $G_{\mathrm{ps}}=2 \pi \times 0.10 \mathrm{~Hz}$ and a corresponding instability threshold power $P_{\text {in }}^{\text {th }}=3.92 \mu \mathrm{W}$ using Eq. (A1). This is in excellent agreement with the theoretical model as it corresponds to an effective coupling and an effective overlap very close to its optimum value $\Lambda=0.95 \Lambda_{\text {opt }}$. The small discrepancy can be explained by the imperfect membrane alignment. The fit for the steady-state beat-note amplitude yields a similar value.

\section{CONCLUSION}

Three-mode cooling and amplification up to the parametric instability have been demonstrated in fully tunable free-space optical cavities. Results demonstrate that the challenging tuning required to enable three-mode interactions can be met in appropriately designed optomechanical systems. The triple resonance allows strong coupling which in turn opens new possibilities for transducers, and single sideband upconverters from the $\mathrm{MHz}$ to the optical band.

Our observation that mechanical mode amplitudes saturate in accordance with our large-amplitude model for parametric instability has interesting implications. It means that in such systems, the saturation acts to control the cavity pump mode power to a constant value independent of the input laser power.

In our system the onset of three-mode instability has not led to loss of cavity lock because the power loss from the $\mathrm{TEM}_{00}$ mode is sufficient to stabilize the system. The work has important implications for large-scale laser interferometer gravitational wave detectors such as Advanced LIGO and Advanced Virgo. First we have confirmed the basic physics of three-mode parametric instability, thereby adding confidence to the prediction that such instabilities will need to be controlled in these detectors. Second, this work shows that instability does not necessarily lead to loss of cavity lock, although it is not possible to directly extrapolate to largescale interferometers which have more complicated control loops and different dynamical range. Further investigation of parametric instability in large interferometers is needed.

\section{ACKNOWLEDGMENTS}

We wish to thank the Gingin Advisory Committee of the LIGO Scientific Collaboration, the LIGO Scientific Collaboration Optics Working Group, and our collaborators Stefan Gossler, Gregg Harry, Stan Whitcomb, and Samuel Deléglise for encouragement and useful advice. This research was supported by Grants No. DP120104676 and No. DP120100898 from the Australian Research Council, by the Agence Nationale de la Recherche program ANR-2011-B504-028-01 MiNOToRe, and by the European FP7 Specific Targeted Research Projects Minos and QNEMS.

\section{APPENDIX A: LARGE-AMPLITUDE MODEL}

Most prior analysis of three-mode interactions have assumed small amplitude [1,2,5,26-28]. While appropriate for obtaining instability criteria, this approach is no longer 


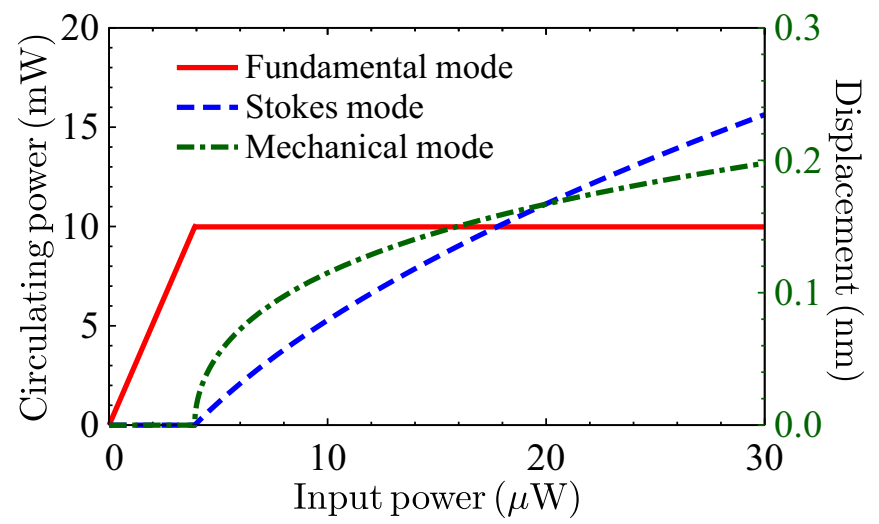

FIG. 7. (Color online) Steady-state behavior of three-mode parametric instability. As soon as the cavity pump circulating power exceeds the instability threshold, the power remains constant for all input powers, while the transverse mode and the mechanical mode amplitude increase monotonically.

relevant once the instability threshold is passed and the loss of fundamental mode power through scattering into highorder mode becomes large. Polyakov and Vyatchanin [18] developed a large-amplitude theoretical model, which is valid for parametric gain $\mathcal{R}>1$. They predicted that the interaction will reach a saturated steady state. If the power is enough to exceed the parametric instability threshold:

$$
P_{\mathrm{th}}=\frac{\hbar \omega_{\mathrm{p}} \gamma_{\mathrm{p}} \gamma_{\mathrm{s}} \Gamma_{\mathrm{m}}}{2 G_{\mathrm{ps}}^{2}},
$$

then the amplitude will grow exponentially. According to Eq. (2.25) of Polyakov and Vyatchanin [18], the initial ring-up rate after turning the laser on will be [29]

$$
\Gamma \approx \frac{2 G_{0 \mathrm{~S}}^{2} P_{\mathrm{in}}}{\hbar \omega_{\mathrm{p}} \gamma_{0} \gamma_{\mathrm{S}}}-\gamma_{\mathrm{m}},
$$

with a coupling strength $G_{i j}$ :

$$
G_{i j}=\frac{2}{L} \sqrt{\frac{\hbar \omega_{i} \omega_{j}}{2 m \Omega_{\mathrm{m}}} \Lambda_{i j}}, \quad(i, j=\mathrm{p}, \mathrm{s}),
$$

where $L$ is the cavity length and $m$ is the effective mass of the mechanical mode. The overlap factor between the optical modes $\hat{a}_{\mathrm{p}}, \hat{a}_{\mathrm{s}}$ and the mechanical mode $\hat{b}_{\mathrm{m}}$ is defined as

$$
\Lambda_{i j}=\left(\int d^{2} \mathbf{r}_{\perp} u\left(\mathbf{r}_{\perp}\right) v_{i}\left(\mathbf{r}_{\perp}\right) v_{j}\left(\mathbf{r}_{\perp}\right)\right)^{2},
$$

where $(i, j=\mathrm{p}, \mathrm{s}) . u$ is the normalized mechanical mode profile, $v_{\mathrm{p}}$ and $v_{\mathrm{s}}$ the normalized optical mode profiles for the pump and scattered modes, and $\mathbf{r}_{\perp}$ is the transverse coordinate on the resonator surface [1].

When the loss of pump mode power through scattering into high-order mode becomes large, the amplitude will stop increasing. According to Eqs. (2.17)-(2.19) of Polyakov and Vyatchanin [18], the steady-state amplitudes of the three-mode system will be (with some change of notation)

$$
\begin{gathered}
\left|a_{\mathrm{p}}\right|^{2}=\frac{\Gamma_{\mathrm{m}} \gamma_{\mathrm{s}}}{G_{\mathrm{ps}}^{2}}, \\
\left|a_{\mathrm{s}}\right|^{2}=\frac{\Gamma_{\mathrm{m}}}{\gamma_{\mathrm{s}}}\left|b_{\mathrm{m}}\right|^{2},
\end{gathered}
$$

$$
\left|b_{\mathrm{m}}\right|^{2}=-\frac{\gamma_{\mathrm{p}} \gamma_{\mathrm{s}}}{G_{\mathrm{ps}}^{2}}+\sqrt{\frac{2 \gamma_{\mathrm{p}} \gamma_{\mathrm{s}}}{\Gamma_{\mathrm{m}} G_{\mathrm{ps}}^{2}} \frac{P_{\mathrm{in}}}{\hbar \omega_{\mathrm{p}}}} .
$$

where $\left|a_{\mathrm{p}}\right|$ is the amplitude of the pump optical mode, $\left|a_{\mathrm{s}}\right|$ is the amplitude of the scattered target high-order mode, and $\left|b_{\mathrm{m}}\right|$ is the amplitude of the mechanical mode of the moving mirror. Figure 7 shows the steady-state amplitude of the cavity mode, the higher-order mode, and the mechanical mode as a function of input power.

\section{APPENDIX B: NUMERICAL SIMULATION OF PARAMETRIC INSTABILITIES}

Here we present the results of our own numerical simulation, which will be used to interpret our experimental results. We assume here that the Stokes mechanism of the three-mode interaction is resonant (i.e., $\Delta \omega=-\Omega_{\mathrm{m}}$ ). To have a better understanding of the whole parametric instability process, we have performed a discrete time-steps simulation. To reduce artifacts, we solve the equations of motion with the Runge-Kutta method [30]. To reduce computation time, we rewrite the equations of motion to have three variables in the following form:

$$
\begin{aligned}
& \dot{a}_{\mathrm{p}}=-\gamma_{\mathrm{p}} a_{\mathrm{p}}+i G_{\mathrm{ps}} a_{\mathrm{s}} e^{i \Omega_{\mathrm{m}} t} \times 2 \operatorname{Re}\left(b_{\mathrm{m}} e^{-i \Omega_{\mathrm{m}} t}\right)+\sqrt{2 \gamma_{\mathrm{p}}} A_{\mathrm{in}}, \\
& \dot{a}_{\mathrm{s}}=-\gamma_{\mathrm{s}} a_{\mathrm{s}}+i G_{\mathrm{ps}} a_{\mathrm{p}} e^{-i \Omega_{\mathrm{m}} t} \times 2 \operatorname{Re}\left(b_{\mathrm{m}} e^{-i \Omega_{\mathrm{m}} t}\right) \\
& \dot{b}_{\mathrm{m}}=-\Gamma_{\mathrm{m}} b_{\mathrm{m}}+i G_{\mathrm{ps}} e^{i \Omega_{\mathrm{m}} t} \times 2 \operatorname{Re}\left(a_{\mathrm{p}}^{*} a_{\mathrm{s}} e^{i \Omega_{\mathrm{m}} t}\right) .
\end{aligned}
$$

The numerical values used for the simulation correspond to the experiment carried out on the near-confocal cavity with intracavity membrane. The initial state is set as

$$
a_{\mathrm{p}}(0)=0, \quad a_{\mathrm{s}}(0)=0, \quad b_{\mathrm{m}}(0)=\sqrt{k_{\mathrm{B}} T /\left(\hbar \Omega_{\mathrm{m}}\right)},
$$

so the system starts with the mechanical resonator thermal noise level and no light resonant in the cavity. In reality, the initial conditions are determined by the thermal distribution of

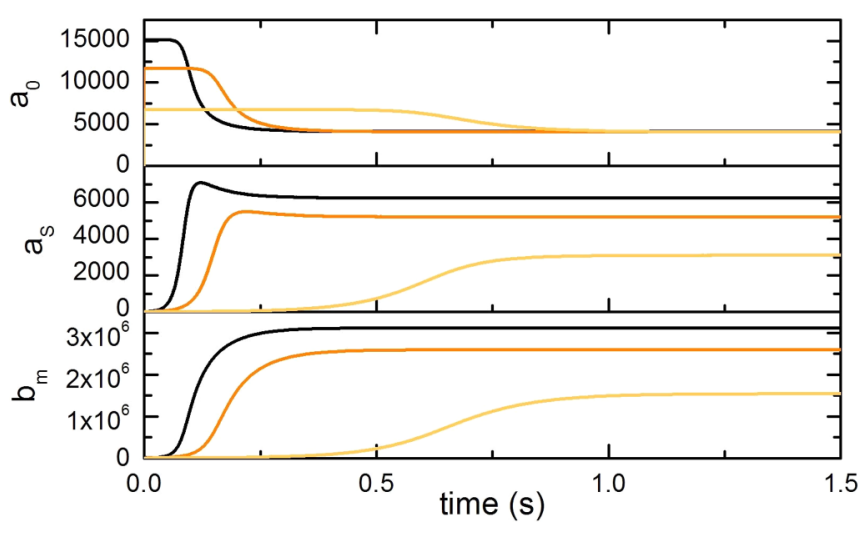

FIG. 8. (Color online) Simulation results for the $\mathrm{TEM}_{00}$ mode amplitude $\left(a_{\mathrm{p}}\right)$, the $\mathrm{TEM}_{20}$ scattered mode amplitude $\left(a_{\mathrm{s}}\right)$, and the mechanical motion amplitude $\left(b_{\mathrm{m}}\right)$. The input powers used here are $5 \mu \mathrm{W}$ [yellow (light gray curves)], $15 \mu \mathrm{W}$ [orange (dark gray curves)], and $25 \mu \mathrm{W}$ (black curves). The system acts to control the pump power in the cavity to a value which is independent of the incident power, by scattering into the mechanical and transverse modes. 
the mode amplitude. Fluctuations in this amplitude (assumed here to have its mean value) lead to small changes on the effective time axis. Because the amplitudes of concern in this paper are large compared with the thermal amplitude, we also neglect the thermal driving of $b_{\mathrm{m}}$ in Eq. (B1).

The simulation results obtained with an input $\mathrm{TEM}_{00}$ power of $5 \mu \mathrm{W}, 15 \mu \mathrm{W}$, and $25 \mu \mathrm{W}$ are shown in Fig. 8. When $a_{\mathrm{p}}$ reaches a threshold value, the parametric amplification process starts and both $a_{\mathrm{s}}$ and $b_{\mathrm{m}}$ start growing. This process goes on until saturation is reached. In the saturation regime, the intracavity pump amplitude $a_{\mathrm{p}}$ is precisely independent of the pump power (see Fig. 8, top curve). The excess optical energy is channeled to the scattered optical amplitude $a_{\mathrm{s}}$ and the mechanical motion amplitude $b_{\mathrm{m}}$. For our parameters, above threshold, the typical scattered circulating power is a few $\mathrm{mW}$ and the typical displacement amplitude of the order of $10^{-10} \mathrm{~m}$ (see Fig. 7).

\section{APPENDIX C: SEMICONFOCAL CAVITY}

\section{Characterization}

A simple characterization setup using a network analyzer, local electrostatic actuation, and a Michelson interferometer to probe the mirror motion allows us to map the vibration profiles and therefore identify the different vibration modes. The corresponding overlap factors $\Lambda_{\mathrm{ps}}$ can then be computed, with Gaussian modes TEM $_{00}$ and TEM $_{04}$ as pump and scattered modes, respectively.

\section{Experiment}

For each set of experiment, we first measure an optical spectrum of the cavity, similar to the one presented in Fig. 11 (for the other experimental setup). This, together with an identification of the transverse mode corresponding to each resonance peak with a CCD camera, allows one to measure the exact optical detuning for a given length of the cavity, which has proven stable over the typical duration of a complete

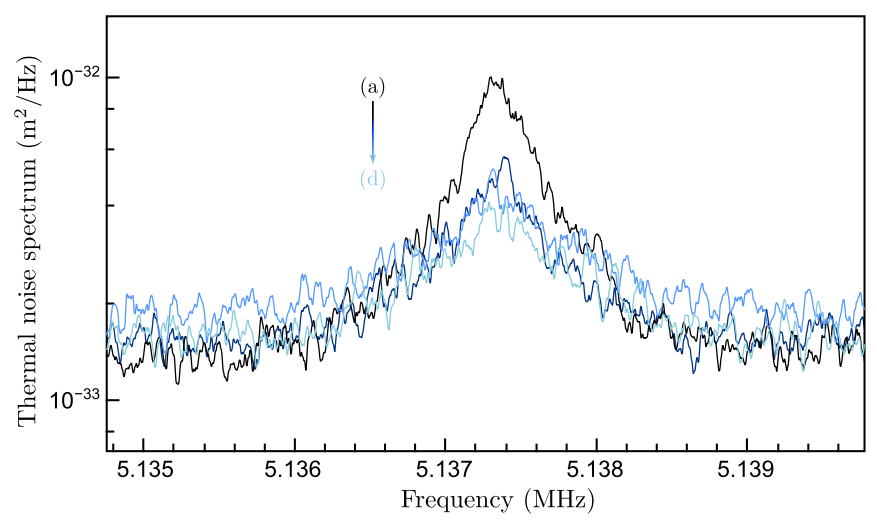

FIG. 9. (Color online) Three-mode cooling. Displacement thermal noise spectra observed close to the $(1,7)$ mechanical resonance frequency, for different frequency offsets $\Delta \omega$ between $\mathrm{TEM}_{00}$ and $\mathrm{TEM}_{04}$ modes of the cavity. Due to the three-mode cooling, the thermal noise spectrum is both widened and reduced. Curves (a)-(d) are measured for different detunings, (a) being the furthest from resonance, and (d) the closest.

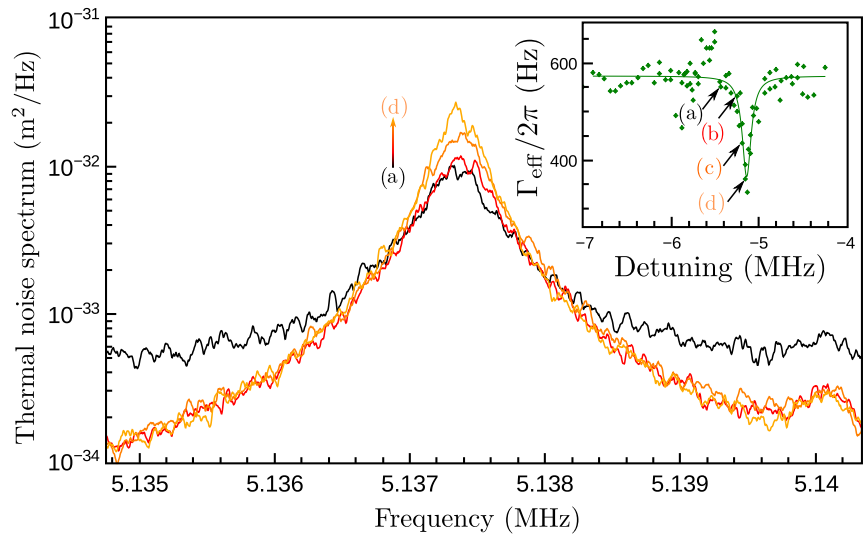

FIG. 10. (Color online) Experimental demonstration of the Stokes mechanism. Thermal noise spectra observed close to the Stokes process resonance. The curves are for different detunings (a)-(d); see insert. (Insert) Evolution of the effective mechanical damping of the $(1,7)$ mechanical mode close to the Stokes resonance condition, together with a fit with theory.

experiment. We then measure the noise spectra for different cavity detunings. Note that because the coating thickness $(\simeq 5 \mu \mathrm{m})$ is not negligible compared to the resonator thickness, the coating process yields tension within and bends the resonator, slightly changing the optical frequency resonance condition.

\section{Experimental results}

Figure 9 presents the typical noise spectra observed for different detunings $\Delta \omega$. As for the usual two-mode cooling experiments, the noise spectrum becomes both wider (as the effective damping $\Gamma$ is increased) and lower (because of the related cold damping effect). Figure 4 of the article presents the measured values of the effective damping, obtained by fitting the noise spectrum. As expected, the damping reaches a peak value at the three-mode resonance (spectrum d) and returns to the intrinsic damping value $\Gamma$ outside the resonance (spectrum a).

Figure 10 presents the results obtained in the opposite case where the Stokes sideband is resonant and amplification occurs. For similar reasons as above, the spectra are both narrower and higher. The insert displays the dependence of the measured effective dampings with the cavity detuning. The same behavior as in the cooling case is observed: maximum effect at the resonance (curve d) and intrinsic damping outside the corresponding cavity bandwidth (curve a).

\section{APPENDIX D: CAVITY WITH MEMBRANE IN THE MIDDLE}

\section{Membrane}

The membrane is a commercial Norcada stoichiometric silicon nitride membrane with tensile stress $T=800 \mathrm{Mpa}$ and density $\rho=2.7 \mathrm{~g} / \mathrm{cm}^{3}$ [31]. It has a square geometry with a side length $D=1 \mathrm{~mm}$ and a thickness $l=50 \mathrm{~nm}$. The effective mass of the membrane is $40 \mathrm{ng}$. The measured resonance frequency is in good agreement with the expected one, given by $\Omega_{i, j} / 2 \pi=\sqrt{T / 4 \rho D^{2}} \sqrt{i^{2}+j^{2}}$. The normalized membrane 
$(i, j)$ mode shape is given as follows $(i, j=1,2, \ldots)[23]$ :

$$
u_{i, j}(x, y)=\frac{2}{D} \sin \left(\frac{i \pi x}{D}+\frac{i \pi}{2}\right) \sin \left(\frac{j \pi y}{D}+\frac{j \pi}{2}\right) \text {. }
$$

\section{Experimental setup}

The optical cavity is mounted on an invar bar in a vibration isolated vacuum tank. Motorized optical mounts and piezoelectric transducers are used for cavity alignment. The Pound-Drever-Hall technique [32] is used to lock the input laser frequency to the cavity $\mathrm{TEM}_{00}$ resonance. We have developed careful alignment and tuning procedures to tune the cavity.

\section{Coupled cavity with a membrane}

The resonance frequency of such a cavity depends on the reflectivity $r$ and relative position of the membrane $z_{0}$ [33]:

$$
\omega_{q}=\omega_{q}^{0}-(c / L) \cos ^{-1}\left[|r| \cos \left(4 \pi z_{0} / \lambda\right)\right],
$$

where $\omega_{q}^{0}$ is the resonance frequency for the corresponding linear cavity, and $r$ is the membrane amplitude reflectivity, which depends on the membrane thickness $l$ and index of refraction $n$ [34] as follows:

$$
r=\frac{\left(n^{2}-1\right) \sin 2 \pi n l / \lambda}{2 i n \cos 2 \pi n l / \lambda+\left(n^{2}+1\right) \sin 2 \pi n l / \lambda} .
$$

In this system, one also has to correct the overlap factors $\Lambda_{i j}$ by an additional dimensionless longitudinal overlap factor $\Lambda_{l}$, which depends on the compound cavity working point and is, when the frequency difference between the target mode and pump mode is much smaller compared with the pump mode $\left|\omega_{\mathrm{s}}-\omega_{\mathrm{p}}\right| \ll \omega_{\mathrm{p}}$, given by

$$
\Lambda_{1}=\sin \left(4 \pi z_{0} / \lambda\right) \sqrt{\frac{|r|^{2}}{1-|r|^{2} \cos ^{2}\left(4 \pi z_{0} / \lambda\right)}} .
$$

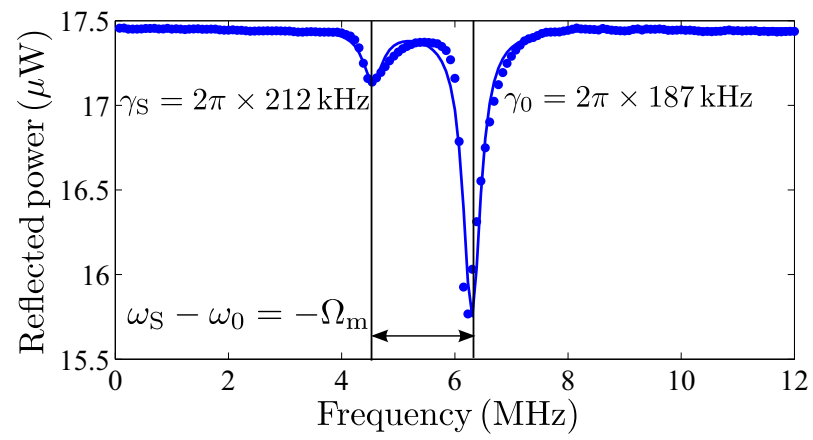

FIG. 11. (Color online) Cavity spectrum when the mode spacing is tuned close to the mechanical mode frequency. A fit with a double Lorentzian allows the frequency difference $\Delta \omega$ and the cavity losses for both optical modes to be determined.

\section{Characterization}

We have measured the dependence of $\mathrm{TEM}_{00}$ and $\mathrm{TEM}_{20}$ mode frequency spacing with the membrane angle. As expected, the frequency spacing is minimized when the membrane is normal to the cavity axis, and varies with a typical rate $\sim 4 \mathrm{MHz} / \mathrm{mrad}$. We have first tuned the membrane position along the optical axis to the desired Crossover 1 position. The optical mode spacing at the avoided crossing is then tuned by membrane angular adjustment. Figure 11 shows the tuned cavity spectrum. We have fitted the spectrum to two Lorentzians to determine the corresponding mode linewidths. As expected, as the optical mode spacing decreases, the cavity mode linewidth increases due to the coupling between the two modes [24]. We continuously monitor the mode shape through the cavity transmission with a CCD camera to ensure the mode shape stays the same. The cavity is stable enough to make repeatable measurements, but retuning is required every day to compensate for very slow drifts.
[1] V. B. Braginsky, S. E. Strigin, and S. P. Vyatchanin, Phys. Lett. A 287, 331 (2001).

[2] V. B. Braginsky, S. E. Strigin, and S. P. Vyatchanin, Phys. Lett. A 305, 111 (2002).

[3] C. Zhao, L. Ju, J. Degallaix, S. Gras, and D. G. Blair, Phys. Rev. Lett. 94, 121102 (2005).

[4] H. S. Bantilan and W. P. Kells, LIGO document LIGO-G070145x0 (LIGO/VIRGO Scientific Collaborations, 2006).

[5] M. Evans, L. Barsotti, and P. Fritschel, Phys. Lett. A 374, 665 (2010).

[6] C. Zhao et al., Phys. Rev. A 78, 023807 (2008).

[7] C. Zhao et al., Phys. Rev. A 84, 063836 (2011).

[8] I. S. Grudinin, A. B. Matsko, and L. Maleki, Phys. Rev. Lett. 102, 043902 (2009).

[9] M. Tomes and T. Carmon, Phys. Rev. Lett. 102, 113601 (2009).

[10] A. B. Matsko, A. A. Savchenkov, V. S. Ilchenko, D. Seidel, and L. Maleki, Phys. Rev. Lett. 103, 257403 (2009).

[11] A. A. Savchenkov, A. B. Matsko, V. S. Ilchenko, D. Seidel, and L. Maleki, Opt. Lett. 36, 3338 (2011).
[12] G. Anetsberger, E. M. Weig, J. P. Kotthaus, and T. J. Kippenberg, C. R. Phys. 12, 800 (2011).

[13] M. E. Tobar and D. G. Blair, J. Phys. D: Appl. Phys. 26, 2276 (1993).

[14] G. M. Harry, Class. Quantum Grav. 27, 084006 (2010).

[15] F. Acernese et al., Class. Quantum Grav. 32, 024001 (2015).

[16] O. Arcizet, P.-F. Cohadon, T. Briant, M. Pinard, and A. Heidmann, Nature (London) 444, 71 (2006).

[17] B. M. Zwickl et al., Appl. Phys. Lett. 92, 103125 (2008).

[18] I. A. Polyakov and S. P. Vyatchanin, Phys. Lett. A 368, 423 (2007).

[19] J. D. Teufel et al., Nature (London) 475, 359 (2011).

[20] J. Chan et al., Nature (London) 478, 89 (2011).

[21] E. Verhagen, S. Deléglise, S. Weis, A. Schliesser, and T. J. Kippenberg, Nature (London) 482, 63 (2012).

[22] O. Arcizet, P.-F. Cohadon, T. Briant, M. Pinard, A. Heidmann, J.-M. Mackowski, C. Michel, L. Pinard, O. Français, and L. Rousseau, Phys. Rev. Lett. 97, 133601 (2006). 
[23] C. Biancofiore, M. Karuza, M. Galassi, R. Natali, P. Tombesi, G. Di Giuseppe, and D. Vitali, Phys. Rev. A 84, 033814 (2011).

[24] T. Klaassen, J. de Jong, M. van Exter, and J. P. Woerdman, Opt. Lett. 30, 1959 (2005).

[25] J. C. Sankey, C. Yang, B. M. Zwickl, A. M. Jayich, and J. G. E. Harris, Nat. Phys. 6, 707 (2010).

[26] L. Ju, S. Gras, C. Zhao, J. Degallaix, and D. G. Blair, Phys. Lett. A 354, 360 (2006).

[27] W. Kells and E. D’ Ambrosio, Phys. Lett. A 299, 326 (2002).

[28] S. E. Strigin and S. P. Vyatchanin, Phys. Lett. A 365, 10 (2007).
[29] X. Chen, Ph.D thesis, University of Western Australian, 2014.

[30] W. H. Press, B. P. Flannery, S. A. Teukolsky, and W. T. Vetterling, Numerical Recipes in FORTRAN: The Art of Scientific Computing (Cambridge University Press, Cambridge, 1992).

[31] S. S. Verbridge, J. M. Parpia, R. B. Reichenbach, L. M. Bellan, and H. G. Craighead, J. Appl. Phys. 99, 124304 (2006).

[32] E. D. Black, Am. J. Phys. 69, 79 (2001).

[33] A. M. Jayich et al., New J. Phys. 10, 095008 (2008).

[34] G. Brooker, Modern Classical Optics (Oxford University Press, Oxford, 2003). 